\title{
The accumulation of podophyllotoxin- $\beta$-D-glucoside by cell suspension cultures derived from the conifer Callitris drummondii
}

Wim van Uden, Niesko Pras, and Theo M. Malingré

University Centre for Pharmacy, Laboratory for Pharmacognosy, University of Groningen, Ant. Deusinglaan 2, 9713 AW Groningen, The Netherlands

Received August 11, 1989/Revised version received June 20, 1990 - Communicated by W. Barz

Due to an unfortunate error, the name of the third author was wrongly spelt. 